\title{
ESTIMATION OF THE CONCENTRATION DISTRIBUTION IN CROSS-FLOW ABSORPTION TOWERS*
}

\author{
YOSHISHIGE HAYASHI, EIJI HIRAI AND HIDEAKI SUEYOSHI** \\ Department of Chemical Engineering, Kanazawa University, Kana- \\ zawa, Japan
}

\begin{abstract}
This paper presents a numerical method based on the Zivi-Brand method applied to crossflow cooling towers and an analytical solution based on the Nusselt solution of heat exchangers for the estimation of the concentration of solute in cross-flow absorption towers. Experimental apparatus used in this work are a wetted-wall tower and a rodlike irrigation tower.

The experimental data for concentration distribution has been obtained for absorption of ammonia in city water in these towers. The results were in good agreement with results calculated by the solution described above. Therefore, these calculation methods are available for the estimation of solute concentration in cross-flow absorption towers.
\end{abstract}

\section{Introduction}

Generally, three different flow patterns are used for gas-liquid contact operations. One is cross flow and the others are countercurrent and cocurrent flows. Cross-flow contact devices have many advantages in the dimensions of equipment, the cost of installation and operating conditions, and they are frequently used for practical absorption operations. In such an absorption tower, since the gas and liquid flow at right angles to each other, the concentrations of solute in both phases change in the directions of flow of the gas and liquid. Therefore, the mechanism of mass transfer in this equipment is considerably more complicated than that in countercurrent- and cocurrent-flow equipment.

Previously, a few solutions for the temperature distribution in cross-flow cooling towers and the performance of cross-flow heat exchangers have been reported by several investigators ${ }^{3 \sim 5)}$. But these solutions have never been confirmed experimentally.

In this paper, a modified Zivi-Brand method and a modified Nusselt solution are derived for the estimation of the concentration distribution. The experimental results for absorption of ammonia in city water in cross-flow absorption towers are compared with the results obtained by the calculation methods described here and the graphical method described in the previous paper ${ }^{2}$. A significant procedure for the design of cross-flow absorption

* Received on March 15, 1971

Presented at the First Autumn Meeting of the Soc. of Chem. Engrs., Japan, Osaka, 1967

** Chiyoda Chemical Engineering and Construction Co., Ltd. towers is presented.

\section{Solution}

The cross-flow absorption tower used in this study is shown schematically as a box with three dimensions $X_{0}, Y_{0}$ and $Z_{0}$, as shown in Fig. 1 .

The liquid flows downward from the top of the tower and the gas flows toward the right. In this case, $X$ and $Y$ denote the axes in the directions of the gas and liquid flows, respectively. To solve this problem, certain simplifying assumptions have been made to reduce the complexity of the equations. These assumptions are as follows:

1) The concentration change of solute along the direction of the tower width (defined as the $Z$-direction) is neglected.

2) The flows of gas and liquid are steady and in plug flow, isothermally.

3) The over-all mass transfer coefficients are constant in the given absorption tower for given conditions.

On the basis of these assumptions, the cross-flow absorption tower may be analyzed mathematically as a two-dimensional mass transfer problem, i.e., the concentration change of solute in the tower may be expressed as a function of the directions of both gas and liquid flows. But the solutions for cross-flow contact devices presented by several investigators ${ }^{3 \sim 5}$ ) cannot be applied to these problems directly. The authors thus have derived a numerical solution and an analytical solution.

\section{Numerical Solution (A Modifid Zivi-Brand Method ${ }^{5)}$ )}

The Zivi-Brand method for the analysis of crossflow cooling towers is a numerical method for deter- 


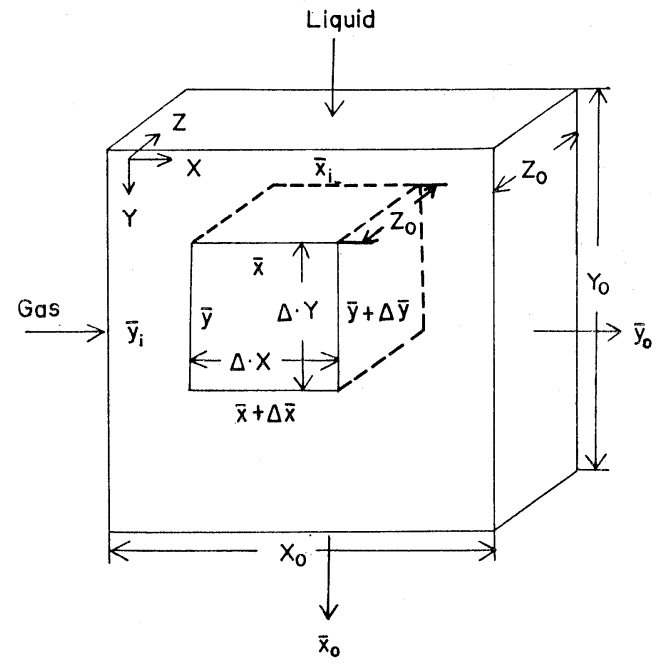

Fig. 1 Schematic model and coordinate system

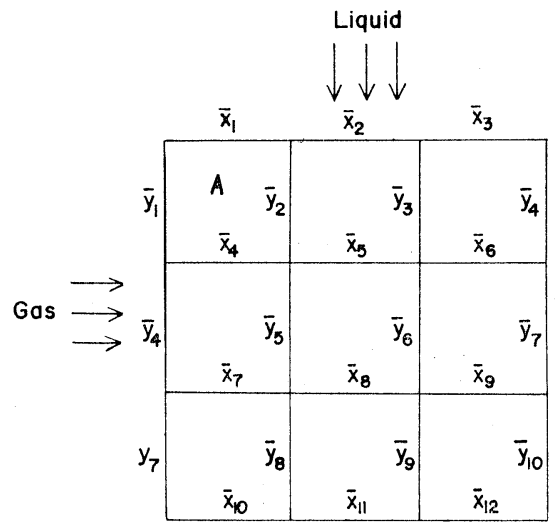

Fig. 2 Representation of the subdivision of absorption tower for the numerical solution

mining temperature contours in the tower by estimating successively the water temperature and the corresponding enthalpy of air at each lattice point in the tower.

Therefore, to calculate the average concentration of solute on a differential element, the Zivi-Brand method may be converted to a form available for this problem. This numerical solution is obtained by solving a two-dimensional differential equation, replacing the partial derivative by a difference. In this case, the rate of mass transfer over a small element of volume of the tower, $\Delta X \cdot \Delta Y \cdot Z_{0}$, is

$$
\begin{aligned}
d N_{A} & =-W_{G} \frac{d Y}{Y_{0}} \frac{\partial \bar{y}}{\partial X} d X=W_{L} \frac{d X}{X_{0}} \frac{\partial \bar{x}}{\partial Y} d Y \\
& =K_{G} a P\left(\bar{y}-\bar{y}^{*}\right) d X d Y Z_{0}
\end{aligned}
$$

From these equations the following is obtained:

$$
\begin{aligned}
& -\frac{W_{G}}{Y_{0}} \frac{\partial \bar{y}}{\partial X}=\frac{W_{L}}{X_{0}} \frac{\partial \bar{x}}{\partial Y} \\
& \frac{W_{L}}{X_{0}} \frac{\partial \bar{x}}{\partial Y}=K_{G} a P\left(\bar{y}-\bar{y}^{*}\right) Z_{0}
\end{aligned}
$$

$\uparrow$ The equilibrium relation is assumed to be a linear function, that is, $\bar{y}^{*}=m_{H} \bar{x}$

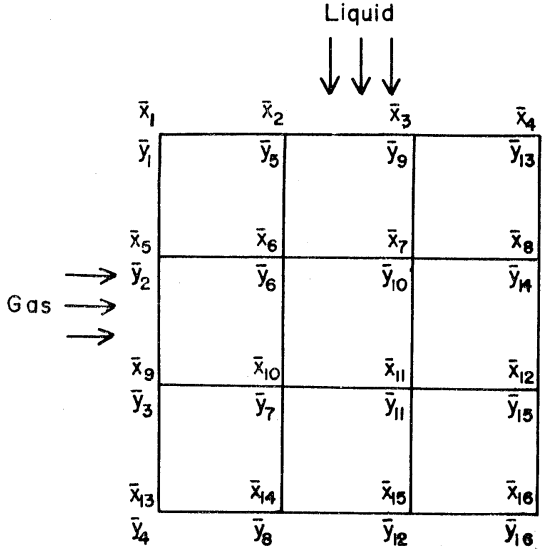

Fig. 3 Representation of the subdivision of absorption tower for the analytical solution

By replacing $\partial \bar{x} / \partial Y$ in Eq.(3) by the finite difference approximation, Eq.(3) becomes

$$
\frac{\Delta \bar{x}}{\Delta Y}=\frac{K_{G} a P\left(\bar{y}-\bar{y}^{*}\right)}{W_{L} / X_{0} Z_{0}}
$$

Now, let us subdivide the dimensions of the tower into several small parts, as shown in Fig. 2, and consider the concentration change of solute in a small element A.

By denoting the concentrations of solute in the liquid phase at the inlet and the outlet of a small element A with $\bar{x}_{1}$ and $\bar{x}_{4}$, respectively, the first difference $\Delta \bar{x} / \Delta Y$ in Eq.(4) is expressed as

$$
\frac{\Delta \bar{x}}{\Delta Y}=\frac{\bar{x}_{4}-\bar{x}_{1}}{\Delta Y}
$$

In addition, $\bar{y}$ and $\bar{y}^{*}$ in a small element $\mathrm{A}$ are assumed to be expressed by the following expressions:

$$
\begin{aligned}
& \bar{y}=\frac{1}{2}\left(\bar{y}_{1}+\bar{y}_{2}\right) \\
& \bar{y}^{*}=\frac{1}{2}\left(\bar{y}_{1} *+\bar{y}_{2} *\right)=\frac{m_{H}}{2}\left(\bar{x}_{1}+\bar{x}_{4}\right)
\end{aligned}
$$

Then, substituting Eqs.(5), (6) and (7) in Eq.(4) gives

$$
\bar{x}_{4}=\frac{\left(1-B m_{H}\right) \bar{x}_{1}+B\left(\bar{y}_{1}+\bar{y}_{2}\right)}{1+B m_{H}}
$$

where $m_{H}$ is a modified Henry constant and $B$ is a dimensionless parameter defined as

$$
B=\frac{\Delta Y K_{G} a P}{2 L / X_{0} Z_{0}}
$$

but $\bar{x}_{4}$ cannot be obtained by Eq.(8), since $\bar{y}_{2}$ is unknown. Then, to eliminate $\bar{y}_{2}$ in Eq.(8), the following mathematical means is used.

By denoting the number of subdivision in two dimensions of the tower with $m$ and $n$, respectively, and rewriting Eq.(2) in terms of the finite difference, it becomes

$$
\frac{\Delta \bar{y}}{\Delta \bar{x}}=-\frac{m}{n} \cdot \frac{W_{L}}{W_{G}}
$$

where $X_{0}=n \Delta X$ and $Y_{0}=m \Delta Y$

In a small element $A$, the concentration difference of solute in the gas phase $\Delta \bar{y}$ is 


$$
\Delta \bar{y}=\bar{y}_{2}-\bar{y}_{1}
$$

To simplify the mathematical manipulation, putting $m=n$ and inserting Eqs.(5) and (11) into Eq. (10), one obtains

$$
\frac{\bar{y}_{2}-\bar{y}_{1}}{\bar{x}_{4}-\bar{x}_{1}}=-\frac{W_{L}}{W_{G}}
$$

Substituting from Eq.(8), one obtains

$$
\bar{x}_{4}=\frac{\left\{1+B\left(W_{L} / W_{G}-m_{H}\right)\right\} \bar{x}_{1}+2 B \bar{y}_{1}}{1+B\left(W_{L} / W_{G}+m_{H}\right)}
$$

and $\bar{y}_{2}$ may be estimated by substituting this equation into Eq.(12). Furthermore, the values of $\bar{y}$ and $\bar{x}$ in other small elements are obtained by carring out the iterative calculation with the generalized difference equations one by one.

Rewriting Eqs.(12) and (13) in the generalized form, they are expressed as follows:

$$
\bar{x}_{k}=\frac{\left\{1+B\left(W_{L} / W_{G}-m_{H}\right)\right\} \bar{x}_{k-w}+2 B \bar{y}_{k-w}}{1+B\left(W_{L} / W_{G}+m_{H}\right)}
$$

and

$$
\bar{y}_{k-w+1}=\bar{y}_{k-w}-\left(W_{L} / W_{G}\right)\left(\bar{x}_{k}-\bar{x}_{k-w}\right)
$$

where $m=n=w, w(w+1) \geqq k \geqq w+1$ and $\bar{y}_{k-w}$ at $X=0$ is $\bar{y}_{i}$,

\section{Analytical Solution ${ }^{1)}$ (A Modified Nusselt Solution $\left.{ }^{4}\right)$}

This analytical solution for cross-flow heat exchangers was presented by Nusselt ${ }^{4}$ and Izumi $^{3}$.

Since this solution is ordinarily applicable to crossflow heat exchangers, it may be applied to cross-flow absorption towers. Therefore, the solution for the evaluation of the concentrations of solute in both phases at each lattice point in the tower is obtained by replacing the temperature and heat transfer coefficient in the differential equation for heat transfer by the concentration of solute and mass transfer coefficient, respectively, and by solving the two-dimensional differential equation with suitable boundary conditions. In this case, to solve this problem, the Laplace transformation method presented by Izumi $^{3)}$ was used. The typical mesh representation of the tower for this problem is shown in Fig. 3. The twodimensional differential equation is

$$
-\frac{W_{G}}{Y_{0}} \frac{\partial \bar{y}}{\partial X}=\frac{W_{L}}{m_{H} X_{0}} \frac{\partial \bar{y}^{*}}{\partial Y}=K_{G} a P\left(\bar{y}-\bar{y}^{*}\right) Z_{0}
$$

and the boundary conditions are

$$
\begin{array}{ll}
\bar{y}=\bar{y}_{i} \text { and } \bar{y}^{*}-\bar{y}_{i}=\left(\bar{y}_{i}{ }^{*}-\bar{y}_{i}\right) e^{-m_{I I} K_{G} a P V Y / W_{L} Y_{0}} \\
\text { at } X=0 \\
\bar{y}^{*}=\bar{y}_{i}{ }^{*} & \text { at } Y=0
\end{array}
$$

To simplify the mathematical manipulations, the following dimensionless terms are introduced:

$$
\begin{array}{lll}
\zeta=\frac{\bar{y}_{i}-\bar{y}^{*}}{\bar{y}_{i}-\bar{y}_{i}{ }^{*}}, & \psi=\frac{\bar{y}_{i}-\bar{y}}{\bar{y}_{i}-\bar{y}_{i}{ }^{*}} \\
\eta=\frac{X}{X_{0}}, & \xi=\frac{Y}{Y_{0}} \\
\alpha=\frac{W_{L}}{m_{H} K_{G} a P V}, & \beta=\frac{W_{G}}{K_{G} a P V}
\end{array}
$$

$\dagger$ In this work, the liquid distributor for the wettedwall tower was the over-flow type, and for the rodlike irrigation tower a trough distributor was used.
Then, Eq.(16) becomes

$$
\zeta-\psi=-\alpha \frac{\partial \zeta}{\partial \xi}=\beta \frac{\partial \psi}{\partial \eta}
$$

and the boundary conditions are rewritten as

$$
\begin{aligned}
& \psi=0 \text { and } \zeta=e^{-\xi / \alpha} \text { at } \eta=0 \\
& \zeta=1 \quad \text { at } \xi=0
\end{aligned}
$$

To solve Eq.(22), applying the two-dimensional Laplace transformation method, one obtains

$$
\begin{aligned}
\zeta(p, q)-\varphi(p, q) & =-\alpha\left\{q \bar{\zeta}(p, q)-\frac{1}{p}\right\} \\
& =\beta p \bar{\psi}(p, q)
\end{aligned}
$$

where $p$ and $q$ are parameters with respect to dimensionless variables $\eta$ and $\xi$, respectively.

Therefore, the solutions of Eq.(25) are

$$
\begin{aligned}
\bar{\zeta}(p, q) & =\frac{\alpha(1+\beta p)}{\beta p+\alpha q(1+\beta p)} \frac{1}{p} \\
\bar{\phi}(p, q) & =\frac{\alpha}{\beta p+\alpha q(1+\beta p)} \frac{1}{p}
\end{aligned}
$$

Then, applying the Heaviside expansion theorem, the inverse transforms of Eqs.(26) and (27) are $\zeta(\eta, \xi)=1-e^{-\eta / \beta}=\left\{\frac{\xi}{\alpha}-\frac{\xi}{2 \alpha^{2}}\left(1-\frac{\eta}{\beta}\right)+\frac{\xi^{3}}{3 ! \alpha^{3}}\left(1-\frac{2 \eta}{\beta}+\frac{\eta^{2}}{2 \beta^{2}}\right)\right.$ $\left.-\frac{\xi^{4}}{4 ! \alpha^{4}}\left(1-\frac{3 \eta}{\beta}+\frac{3}{2} \frac{\eta^{2}}{\beta^{2}}-\frac{\eta^{3}}{6 \beta^{3}}\right)+\cdots \cdot \cdots\right\}$

and

$$
\begin{aligned}
& \psi(\eta, \xi)=1-e^{-\eta / \beta}\left\{1+\frac{\eta \xi}{\alpha \beta}-\frac{\xi^{2}}{4 \alpha^{2}}\left(\frac{2 \eta}{\beta}-\frac{\eta^{2}}{\beta^{2}}\right)\right. \\
& +\frac{\xi^{3}}{3 ! \alpha^{3}}\left(\frac{\eta}{\beta}-\frac{\eta^{2}}{\beta^{2}}+\frac{\eta^{3}}{6 \beta^{2}}\right)-\frac{\xi^{4}}{4 ! \alpha^{4}}\left(\frac{\eta}{\beta}-\frac{3}{2} \frac{\eta^{2}}{\beta^{2}}\right. \\
& \left.\left.+\frac{\eta^{3}}{2 \beta^{3}}-\frac{1}{24} \frac{\eta^{4}}{\beta^{4}}\right)+\cdots \cdots \cdots\right\}
\end{aligned}
$$

where, $0 \leqq \eta \leqq 1$ and $0 \leqq \xi \leqq 1$.

\section{Experimental Equipment and Instrumentation}

Fig. 4 shows the dimensions and diagrams of experimental absorption towers used in this work. One of these towers is a wetted-wall tower and the other is a rod-like irrigation tower. These towers are rectangular boxes of $400 \times 400 \times 100 \mathrm{~mm}^{3}$, and are made of polyvinyl chloride plates. The inside wall of the wetted-wall tower is lined with a thin polyvinyl chloride plate, the surface of which is treated with hydrophilic chemicals to provide extensive interfacial area in the tower.

The liquid was heated in storage to the same temperature as the mixed gas, then distributed at the top of the tower by a liquid distributor ${ }^{\dagger}$ and flowed uniformly downward throughout the tower.

Air from a blower was almost saturated by cross-flow contact with water in a packed tower, then sent to the homogenizing chamber and mixed with solute gas from a cylinder. Air and solute gas flow rates were measured by a hot-wire anemometer and a capillary-tube flowmeter, respectively. The mixed gas, whose flow was ordered by a row of parallel plates with respect to the axis of its duct, was blown into the side inlet of the tower and came into contact with the liquid flow at right angles in the tower. 
Table 1 The calculated values of the concentration of solute in the liquid phase at the bottom of the tower

$$
\begin{aligned}
& \text { Volume of a small } \\
& \text { element }
\end{aligned}
$$

$m=n=3 \quad 133 \times 133 \times 100 \mathrm{~mm}^{3}$ $m=n=5 \quad 80 \times 80 \times 100 \mathrm{~mm}^{3}$ $m=n=10 \quad 40 \times 40 \quad 100 \mathrm{~mm}^{3}$
Eqs. (13) Eq.(28) and (15)

$0.00512 \quad 0.00581$

$0.00507 \quad 0.00550$

$0.00506 \quad 0.00527$

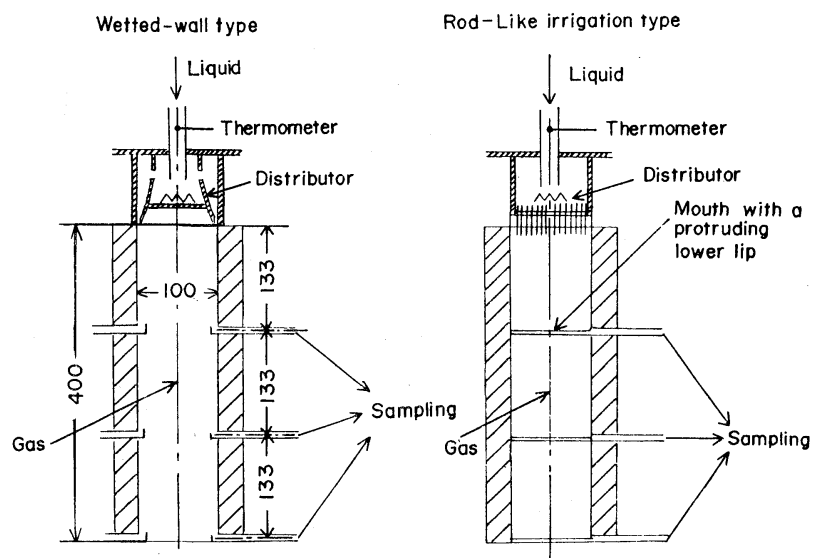

Fig. 4 Vertical section through experimental apparatus (absorption towers)

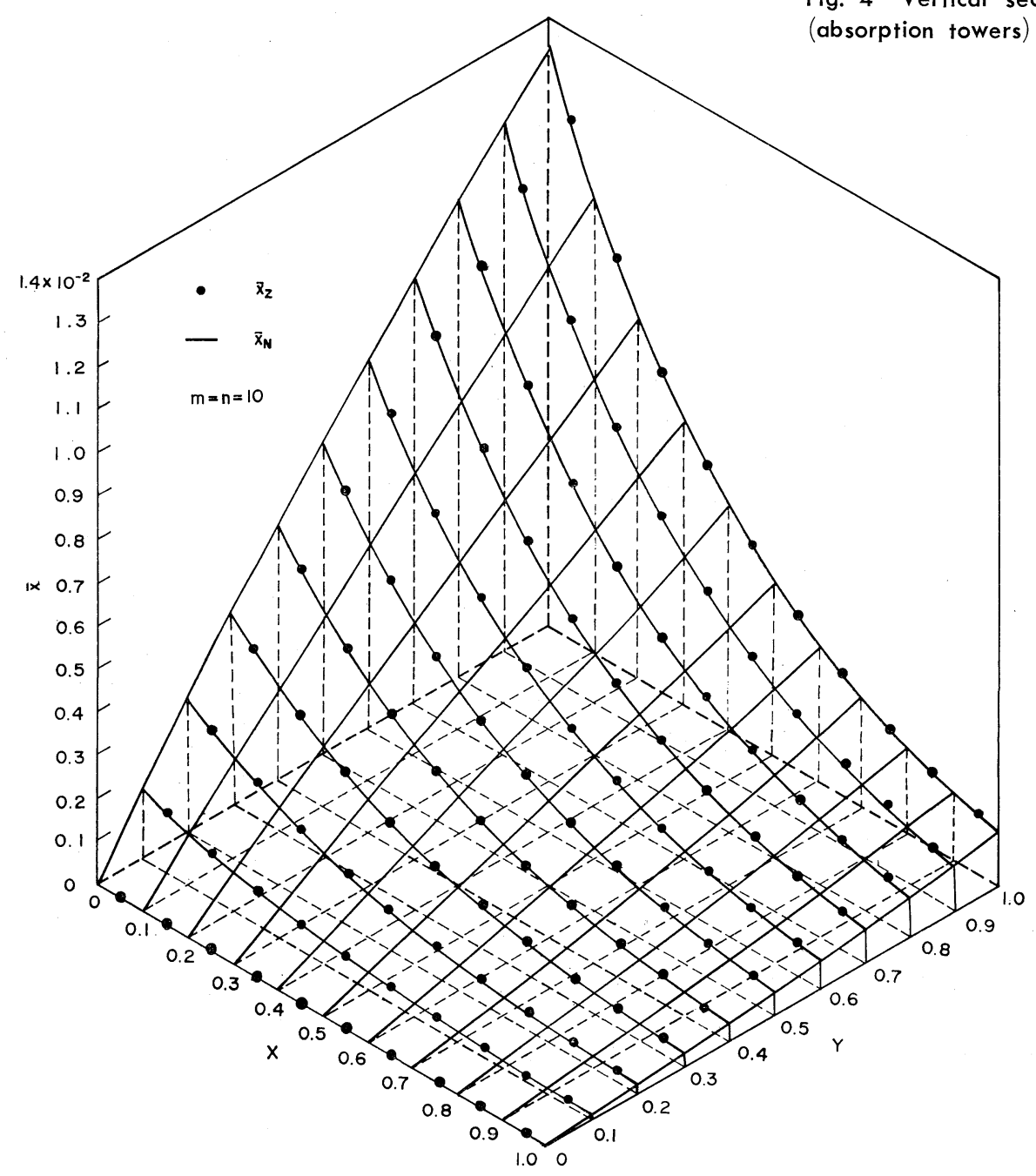

$\bar{x}_{Z}:$ the results calculated by Eqs. (14) and (15)

$\bar{x}_{N}:$ the results calculated by Eq. (28)

Fig. 5 Calculated values of a numerical example (where $m=n=10$ )

The inlet-mixed gas temperatures were measured with a thermistor. The chamber and the duct were covered with asbestos plate to avoid the condensation of vapor. In this experiment, to simplify calculation, the dimensions of the tower were subdivided into three equal parts of $133 \times 133 \times 100 \mathrm{~mm}^{3}$, and the air $-\mathrm{NH}_{3}-$ water system was used.

The experiment was carried out at atmospheric pressure, and the concentration of solute in the liquid phase at each lattice point was determined by the titration method with $0.2 \mathrm{~N} \mathrm{H}_{2} \mathrm{SO}_{4}$ solution.

\section{Experimental Results and Discussion}

Distribution of concentrations. The application of these calculation methods to a practical case is illustrated by the following numerical example. The operating conditions and data are listed below: 
Fig. 6 Concentration profile of solute in the liquid phase (where $\bar{x}_{Z}$ and $\bar{x}_{N}$ are the same as those in Fig. 5 and $\bar{x}_{G}$ are the estimated values by graphical method.)
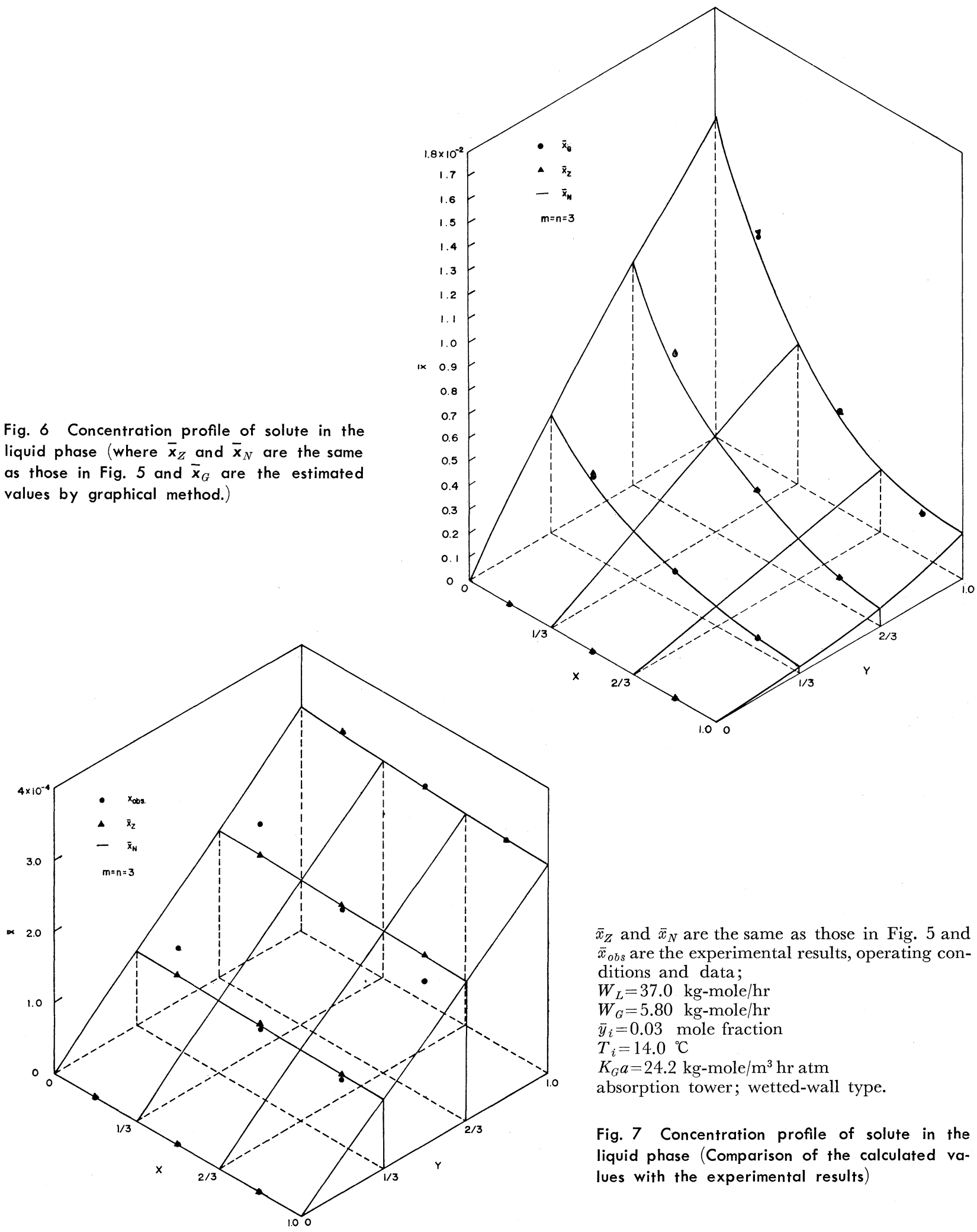

$\bar{x}_{Z}$ and $\bar{x}_{N}$ are the same as those in Fig. 5 and $\bar{x}_{\text {obs }}$ are the experimental results, operating conditions and data;

$W_{L}=37.0 \mathrm{~kg}-\mathrm{mole} / \mathrm{hr}$

$W_{G}=5.80 \mathrm{~kg}-\mathrm{mole} / \mathrm{hr}$

$\bar{y}_{i}=0.03$ mole fraction

$T_{i}=14.0{ }^{\circ} \mathrm{C}$

$K_{G} a=24.2 \mathrm{~kg}-\mathrm{mole} / \mathrm{m}^{3} \mathrm{hr}$ atm

absorption tower; wetted-wall type.

Fig. 7 Concentration profile of solute in the liquid phase (Comparison of the calculated values with the experimental results)

$$
\begin{aligned}
& W_{L}=47.0 \\
& W_{G}=5.20 \\
& \bar{y}_{i}=0.05 \\
& T_{i}=23.2 \\
& \bar{x}_{i}=0 \\
& \bar{x}_{0}=5.04 \times 10^{-3}
\end{aligned}
$$

$$
\begin{aligned}
& m_{H}=1.02 \\
& K_{G} a=917 \\
& \text { Packing material: 3/4" Raschig rings }
\end{aligned}
$$

where $K_{G} a$ was calculated by the correction factor method which had been reported by authors ${ }^{2)}$ under these conditions and data. 


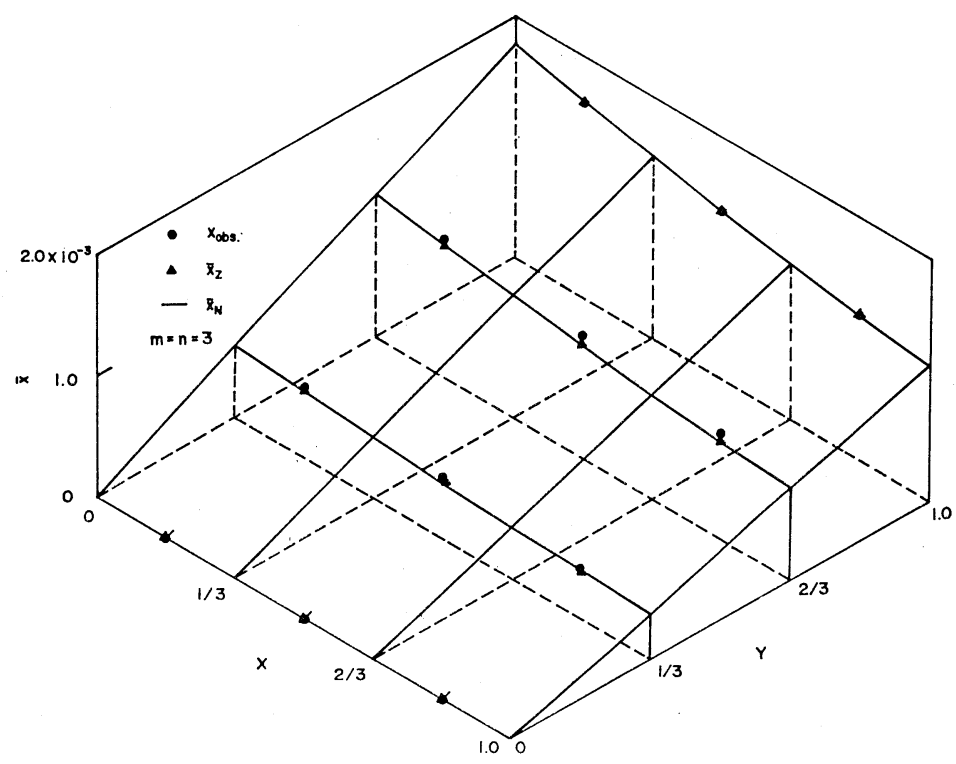

$\bar{x}_{Z}, \bar{x}_{N}$ and $\bar{x}_{\text {obs }}$ are the same as those in

Fig. 7, operating conditions and data;

$W_{L}=47.0 \mathrm{~kg}-\mathrm{mole} / \mathrm{hr}$

$W_{G}=6.00 \mathrm{~kg}-\mathrm{mole} / \mathrm{hr}$

$\bar{y}_{i}=0.03$ mole fraction

$T_{i}=15.4{ }^{\circ} \mathrm{C}$

$K_{G} a=175 \mathrm{~kg}-\mathrm{mole} / \mathrm{m}^{3} \mathrm{hr}$ atm

absorption tower; rod-like irrigation type.

Fig. 8 Concentration profile of solute in the liquid phase (Comparison of the calculated values with the experimental results)

The cross-flow packed absorption tower used in this example had the same dimensions as the rodlike irrigation tower described here.

The concentrations of solute in both phases are calculated by Eqs.(14), (15), (28) and (29). The calculations were performed by means of a NEAC 2230 digital computer. The calculated result for $m=n$ $=10$ is shown in Fig. 5. In addition, the calculated values of the average concentration of solute in the liquid phase at the outlet for $m=n=3,5$ and 10 are summarized in Table 1 . The experimental result for this case was 0.00504 . This result is consistent with the calculated values shown in Table 1 . As is evident from Table 1, the values calculated approach each other closely as $m$ and $n$ increase, i.e., the truncation error in the solutions may be ignored.

So long as the equilibrium relation is approximately linear over the range of composition, the calculation methods described in this paper are available for the evaluation of the concentration distribution of solute in the tower. However, the equilibrium curves are not strictly straight over an extensive range of compositions. Therefore, these calculation methods are restricted to the linear portion of the equilibrium curves.

Previously, to obtain the concentration distribution of solute in cross-flow absorption towers, the authors reported a graphical method ${ }^{2)}$ which is appropriate to this problem over an extensive range of equilibrium curves.

Fig. 6 shows a comparison of the values calculated by these methods and the results estimated by the graphical method. It shows that $\bar{x}_{N}$ and $\bar{x}_{Z}$ are considerably consistent with $\bar{x}_{G}$.

Figs. 7 and 8 show the experimental results for the

$\dagger$ These contours are determined from the concentration of lattice point in the tower by means of interpolation formula. absorption of ammonia in city water in a wetted-wall tower and a rod-like irrigation tower and the values calculated by Eqs.(14), (15) and (29). These experimental results are the average values over the interval of adjacent measured points in the tower. Some of them are slightly dispersed due to inadequate liquid distribution. However, these experimental data agree well with the calculated results. Therefore, it is thought that these calculation methods are fairly appropriate for the estimation of concentration distribution in absorption towers.

\section{Design of Cross-Flow Absorption Towers}

The calculation methods described here can be put into a form available for the design of cross-flow absorption towers. To simplify these calculations, it is advisable to express the coordinates of the grid in dimensionless form. Then the coordinates are introduced in terms of the following dimensionless variables :

$$
\begin{aligned}
& X_{L}=X_{0} \frac{K_{G} a P}{W_{G} / A_{G}} \\
& Y_{L}=Y_{0} \frac{K_{G} a P}{W_{L} / A_{L}}
\end{aligned}
$$

Contours ${ }^{\dagger}$ of constant liquid concentration in the tower for $\bar{y}_{i}=0.01$ mole fraction and $T_{i}=22^{\circ} \mathrm{C}$ are shown in Fig. 9. The dimensionless coordinates of Fig. 9 can be applied to the calculation for any cross-flow absorption towers for which $K_{G} a, W_{L}, W_{G}$, $X_{0}, Y_{0}$ and $Z_{0}$ are known. For example, when $K_{G} a=$ $300 \mathrm{~kg}-\mathrm{mole} / \mathrm{m}^{3} \mathrm{~atm} \mathrm{hr}, W_{G} / A_{G}=120 \mathrm{~kg}-\mathrm{mole} / \mathrm{m}^{2} \mathrm{hr}$, $W_{L} / A_{L}=600 \mathrm{~kg}-\mathrm{mole} / \mathrm{m}^{2} \mathrm{hr}$ and $X_{0}=Y_{0}=40 \mathrm{~cm}$ from Eqs. (30) and (31), one obtains $X_{L}=1.0$ and $Y_{L}=0.2$. For this example, the outlet concentration of the absorption tower would be represented by the heavy line in Fig. 9.

Usually the engineer is interested in the average concentration of solute in the liquid phase leaving 
Fig. 9 Concentration contours for $\bar{y}_{i}=$ 0.01 mole fraction and $T_{i}=22^{\circ} \mathrm{C}$
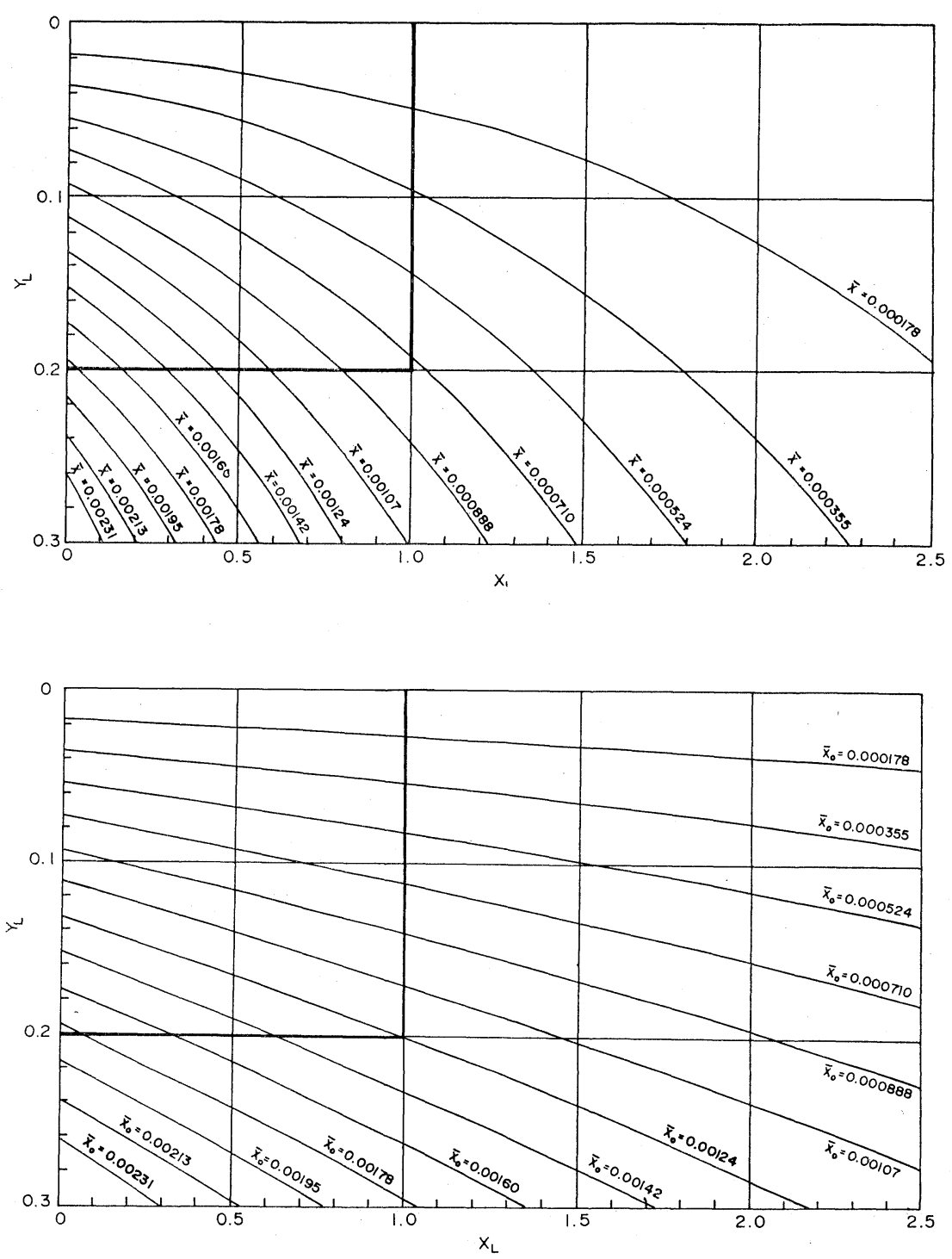

Fig. 10 Contours of exit mean concentrations for $\bar{y}_{i}=0.01$ mole fraction and $T_{i}=22^{\circ} \mathrm{C}$ the tower for the design of absorption towers. The data of Fig. 9 were used to calculate exit mean concentrations. The results are shown in Fig. 10. At any point in the grid, the concentration indicated refers to the average value over the bottom of the tower. For example, in Fig. 10 the average concentration shown at $X_{L}=1.0, Y_{L}=0.2$ is 0.00124 mole fraction. This means that a tower having a dimensionless height, $Y_{L}$, of 0.2 , and a dimensionless depth, $X_{L}$, of 1.0, would discharge an aqueous ammonia solution of 0.00124 mole fraction when the process is operating at $\bar{y}_{i}=0.01$ mole fraction and $T_{i}=22^{\circ} \mathrm{C}$. Therefore, the contour charts of exit mean concentrations are applicable to estimate $K_{G} a$ or the dimensions of the tower.

1) When $X_{0}, Y_{0}, Z_{0}, W_{L}, W_{G}$ and $\bar{x}_{0}$ are known, from Eqs.(30) and (31), one obtains

$$
\frac{X_{L}}{Y_{L}}=\frac{X_{0}}{Y_{0}} \frac{W_{L} / A_{L}}{W_{G} / A_{G}}
$$

where $X_{L}$ and $Y_{L}$ are determined by the point of intersection of the straight line which represents the linear relationship of Eq.(32) and the curves of $\bar{x}_{0}$. Then $K_{G} a$ is estimated by the above values of $X_{L}$ and
$Y_{L}$ decided graphically.

2) When $K_{G} a, W_{L} / A_{L}, W_{G} / A_{G}$ and $X_{0} / Y_{0}$ are chosen arbitrarily and $\bar{x}_{0}$ is determined, the dimensions of the tower are estimated by the values of $X_{L}$ and $Y_{L}$ determined by a method similar to that mentioned above.

Thus the contour charts are useful for estimating practical tower size.

\section{Conclusion}

The numerical solution and the analytical solution for the concentration of solute in cross-flow absorption towers were studied. The results have been presented in such a form that tower dimensions can be calculated when the over-all volumetric mass transfer coefficient is known. The experimental results agree well with the values calculated by Eqs.(14), (15), (28) and (29) and the graphical method described in the previous paper ${ }^{2)}$. However, according to our theoretical considerations, the graphical method is not so available for the special cases of absorption accompanied by heat generation due to the reaction, large values of the ratio of liquid 
flow rate to gas flow rate, and extremely small change of compositions in both phases, because the accuracy of $\bar{y}^{*}$ is not satisfactory. In addition, this method usually requires a tedious procedure. Therefore, so long as the equilibrium relation is approximately linear, the modified Zivi-Brand method and the modified Nusselt solution, which do not require tedious calculations, are sufficiently available for the evaluation of the concentration of solute in both phases. On the other hand, when the equilibrium curves cannot be approximated by a linear function with respect to compositions, the graphical method is applicable to the practical absorption problem of crossflow towers except for some special cases.

\section{Nomenclature}

$A_{G}=$ cross sectional area of tower at right angles to gas flow

$A_{L}=$ cross sectional area of tower at right angles to liquid flow

$\left[\mathrm{m}^{2}\right]$

$a=$ effective surface area per unit volume

$B=$ dimensionless parameter defined by Eq. (9)

$\left[\mathrm{m}^{2} / \mathrm{m}^{3}\right]$

$K_{G}=$ over-all mass transfer coefficient in gas phase

[kg-mole $/ \mathrm{m}^{2} \mathrm{hr}$ atm]

$m=$ number of partition in space along $Y$

$m_{H}=$ the modified Henry constant

$N_{A}=$ rate of mass transfer

$n=$ number of partition in space along $X$

$P \quad=$ total pressure

$p=$ a parameter with respect to $\eta$

$q=$ a parameter with respect to $\xi$

[kg-mole/hr]

$[-]$

[atm]
$T=$ local liquid temperature

$V=$ effective tower volume

$W_{G}=$ average gas flow rate

$W_{L}=$ average liquid flow rate

$X=$ horizontal coordinate (gas flow direction)

$X_{L}=$ dimensionless variable, defined by Eq.(30)

$X_{0}=$ effective tower depth

$\bar{x}=$ local concentration of solute gas in liquid phase (mole fraction)

$Y=$ vertical coordinate (liquid flow direction)

$Y_{L}=$ dimensionless variable, defined by Eq.(31)

$Y_{0}=$ effective tower height

$\bar{y}=$ local concentration of solute gas in gas phase (mole fraction)

$\bar{y}^{*}=$ local concentration of $\bar{y}$ equilibrium with $\bar{x}$ (mole fraction)

$Z_{0}=$ effective tower width

$\alpha=L / m_{H} K_{G} a P V$, defined by Eq. (21)

$\beta=G / K_{G} a P V$, defined by Eq.(21)

$\zeta, \psi=$ concentration ratio, defined by Eq. (19)

$\eta, \xi=$ dimensionless length, defined by Eq.(20)

〈Subscripts〉

$\mathrm{i}=$ inlet mean

$\mathrm{o}=$ outlet mean

$1,2,3 \ldots \ldots \ldots 16=$ position number, reference to Figs. 2 and 3

\section{Literature Cited}

1) Hayashi, Y. and E. Hirai: Kagaku Kōgaku, 34, 965 (1970)

2) Hayashi, Y. and E. Hirai: ibid., 35, 214 (1971)

3) Izumi, R.: Memoirs of the Faculty of Technology, Yamagata University, No. 2, 412 (1951)
4) Nusselt, W.: Tech. Mech. Thermodynam., 1, 417 (1940).

5) Zivi, S. M. and B. B. Brand: Refrigerating Eng., 8, 31 (1956)

\title{
THE ELUTRIATION RATE FROM A PACKED FLUIDIZED BED*
}

\author{
KUNIO KATO AND UTARO ITO \\ Department of Chemical Engineering, Gunma University, Kiryu, Japan
}

The particle elutriation rate from a packed fluidized bed consisting of particles of almost uniform size is measured for various reduced bed heights, $L_{m f} / L_{T}$ various reduced gas velocities $U / U_{T}$, and various packing sizes. An experimental equation for that rate is obtained.

The elutriation rate of fine particles from a packed fluidized bed comprising particles of two sizes is also measured under various experimental conditions. That rate is proportional to the concentration of fine particles in the bed. An experimental equation for the elutriation rate constant is obtained

Finally, the mechanism of elutriation from the packed fluidized bed is discussed.

\section{Introduction}

In designing a particle-fluid reactor, such as a fluidized bed or a packed fluidized bed, it is quite important to estimate the particle elutriation rate from the column in order to determine the operating

* Received on June 17, 1971

Presented at the 36th Annual Meeting of the Soc. of Chem. Engrs., Japan, April 4, 1971 range of gas flow rate and to design a suitable particle collector. The elutriation from the fluidized bed has been studied by Leva1), Wen et al.4), Osberg et al. ${ }^{2)}$, Zenz et al. ${ }^{6)}$, Yagi et $a l .{ }^{5}$ ) and Tanaka et $a l .{ }^{3)}$ But the elutriation from the packed fluidized bed has been little studied.

In this study, the elutriation rates from a packed fluidized bed consisting of particles of almost uniform size or particles of two sizes are measured under various operating conditions. Experimental equations 